\title{
Programa de autorregulación emocional para el pensamiento crítico y creativo en estudiantes de la institución educativa 821563-Gallito Ciego
}

\author{
Mg. Clariza Ely Romero Mendoza \\ rmendozace@ucvvirtual.edu.pe \\ https://orcid.org/0000-0003-0835-9803 \\ Programa Académico de Doctorado en Educación \\ Escuela de postgrado \\ Universidad César Vallejo \\ Chiclayo - Perú
}

\section{RESUMEN}

Esta investigación tuvo como objetivo determinar el grado de influencia del programa de autorregulación emocional en el desarrollo del pensamiento crítico y creativo de los estudiantes de la Institución Educativa 821563-Gallito Ciego-Cajamarca. El tipo de estudio es básico, propositiva y diseño no experimental de corte transversal; la muestra fueron 35 estudiantes de primero al sexto grado y 3 docentes de la Institución Educativa No 821563 Gallito Ciego; se usaron como instrumentos el cuestionario, la guía de entrevista y la guía de observación. Se diseñó un programa de autorregulación emocional para el desarrollo del pensamiento crítico y creativo en estudiantes de la Institución Educativa 821563-Gallito Ciego; el mismo que permitirá a partir del control de las emociones y madurez emocional el desarrollo del pensamiento crítico y creativo en los estudiantes. Mediante su aplicación, se conseguirá que los estudiantes mejoren su inteligencia desde sus habilidades de pensamiento crítico, creando espacios donde prevalezcan la crítica, la reflexión, la creatividad y el análisis, a partir del desarrollo de la autorregulación emocional; el mismo se validó a juicio de 5 expertos.

Palabras clave: autorregulación emocional; pensamiento crítico; creatividad. 
Programa de autorregulación emocional...

\title{
GeoGebra for the development of critical and creative thinking in secondary school students
}

\begin{abstract}
This research aimed to determine the degree of influence of the emotional self-regulation program on the development of critical and creative thinking of students of the Educational Institution 821563-Gallito Ciego-Cajamarca. The type of study is basic, propositional and non-experimental design cross-sectional; the sample were 35 students from first to sixth grade and 3 teachers from the Educational Institution $N^{\circ} 821563$ Gallito Ciego; were used as instruments the questionnaire. An emotional self-regulation program was designed for the development of critical and creative thinking in students of the Educational Institution 821563-Gallito Ciego; the same that will allow from the control of emotions and emotional maturity the development of critical and creative thinking in students. Through its application, students will be able to improve their intelligence through their critical thinking skills, creating spaces where criticism, reflection, creativity and analysis prevail, based on the development of emotional self-regulation; It was validated by 5 experts.
\end{abstract}

Keywords: emotional self-regulation; critical thinking; creativity

Artículo recibido: 30 noviembre. 2021 Aceptado para publicación: 29 diciembre 2021 Correspondencia: rmendozace@ucvvirtual.edu.pe Conflictos de Interés: Ninguna que declarar 


\section{INTRODUCCIÓN}

En la actualidad y frente al mundo globalizado existe la preocupación de lograr la calidad educativa considerando todos sus elementos, que hacen propicio el desarrollo de la educación, siendo entonces fundamental que en la formación del estudiantado se tenga en cuenta el crecimiento de los elementos neurofisiológicos, así como producción de estrategias para que los estudiantes manejen juicos críticos en los diferentes escenarios de actuación.

En América Latina, la formación del pensamiento crítico y creativo es tema de interés desde años atrás y las investigaciones al respecto siguen teniendo vigencia, asimismo, existen autores que mencionan que las emociones se encuentran particularmente ligadas al pensamiento crítico y creativo. Sin embargo, los programas de desarrollo del pensamiento crítico no se han implementado de forma general en todas instituciones educativas, o, en caso han sido implementadas, éstas no han surgido el efecto esperado. Conforme al Estudio Regional Comparativo y Explicativo elaborado por la Unesco (2019), evidenció que en la República argentina es necesario la promoción del pensamiento crítico para que los alumnos sean capaces de hallar soluciones a las distintas problemáticas que enfrentan diariamente, ya que en pleno siglo XXI, estas son habilidades de suma importancia.

El Programa Internacional para la Evaluación de Estudiantes informa que el Perú se ubica con un bajo promedio de desempeño en comprensión lectora y resolución de problemas (Ministerio de Educación, 2015a) habiendo creado las rutas del aprendizaje donde señala que el pensamiento crítico, de la emisión de divergentes juicios, la reflexión sobre distintas materias, la extracción de conclusiones, el análisis crítico y la distinción de ideas de forma autónoma temas capacidad de reflexionar, emitir juicios divergentes, extraer conclusiones, analizar críticamente y distinguir ideas de manera autónoma es primordial para superar las deficiencias relacionadas con la lectura y resolución de problemas orientadas al desarrollo del pensamiento crítico y creativo.

La región de Cajamarca en el 2019, muestra bajos resultados en la prueba pisa a nivel nacional, a pesar de tener más colegios con jornada escolar completa y mayor número de docentes. En el área de matemática, solo 26 de cada 100 estudiantes muestra capacidad para la resolución de una problemática planteada, mientras que solo 27 son capaces de comprender lo que leen, por debajo del promedio nacional (Sineace, 2020). Es por ello, que las instituciones educativas ejerciten la formación del pensamiento crítico a lo largo 
del procedimiento de aprendizaje - enseñanza, con la finalidad de que el estudiantado afiance sus actitudes críticas. En la Institución Educativa 821563 de Gallito CiegoCajamarca, hay 35 estudiantes de primer a sexto año del nivel primario; se llegó a advertir en el estudiantado marcadas deficiencias respecto al afianzamiento del pensamiento crítico y creativo, los estudiantes generalmente no hacen enjuiciamientos, no cuestionan a la realidad la información que obtienen, aceptándolas tal como se las recibe, siendo mayormente receptores, asimismo no muestran actitudes de hacer creaciones, innovaciones de hechos, contenidos, aspectos que se tratan durante el despliegue de las clases de aprendizaje, limitadas situaciones de trabajo sobre autorregulación emocional con los estudiantes relacionadas con el grado y nivel de estudios.

\section{ESTRATEGIAS METODOLÓGÍCAS}

\subsection{Tipo y diseño de investigación}

\subsubsection{Tipo de investigación: Básica.}

Está direccionada a lograr nuevos saberes sistemáticamente, con el único objetivo de lograr un incremento en los conocimientos de un contexto concreto (Álvarez, 2020). La investigación es una mezcla de razonamiento y experiencia y puede considerarse que es la manera más pertinente para arribar al descubrimiento de lo verdadero, en especial en las ciencias naturales.

\subsubsection{Diseño de investigación}

Es propositiva porque es un proceso que emplea la dialéctica utilizando una variedad de procedimientos y técnicas con el propósito de llegar a un diagnóstico y resolución de resolver problemas básicos, para hallar respuestas a preguntas preparadas científicamente, estudiando las relaciones entre factores y eventos o la formación de conocimientos de característica científica (Giler, 2018). El diseño de investigación es no experimental de corte transeccional; no se llevó a cabo la manipulación de las variables por el lado del sujeto que realiza la investigación, se mide una sola vez las variables y con esa información se realiza el análisis (Álvarez, 2020). Es de corte transeccional, dado que, los datos serán recopilados en un momento solo, en único tiempo, en un menor periodo al de un año dado que la finalidad del estudio es la descripción de los productos y, de esa manera llevar a cabo el análisis y la posible relación entre las variables investigadas. 


\section{Figura 1}

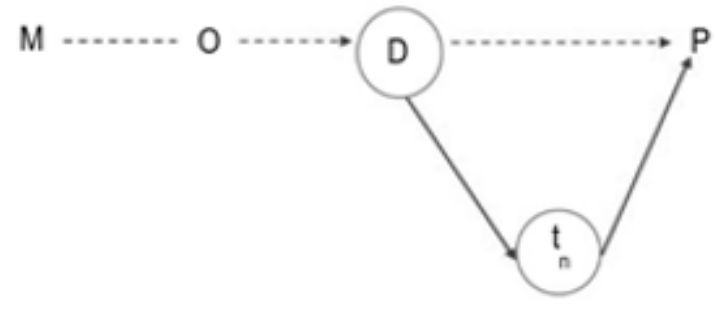

Donde:

M = Muestra de estudio conformado por los estudiantes de primero al sexto grado de la Institución Educativa No 821563 Gallito Ciego.

$\mathrm{O}=$ Información relevante recogida de los participantes en la investigación en relación a las variables de estudio.

D = Diagnóstico y evaluación

Tn = Análisis y fundamentación teórica.

$\mathrm{P} \quad=$ Producto (Programa de autorregulación emocional).

\subsection{Variables y operacionalización}

\subsubsection{Variable 1 y/o independiente: Autorregulación emocional.}

\subsubsection{Variable 2 y/o dependiente: Pensamiento crítico y creativo.}

\subsubsection{La operacionalización de las variables se encuentra ubicada en el anexo 01.}

\subsection{Población, muestra, muestreo}

\subsubsection{Población}

El universo poblacional en la presente investigación estuvo constituido por $\mathbf{3 5}$ estudiantes del primero a sexto grado de estudios y por 3 docentes de la institución educativa $\mathrm{N}^{\mathrm{o}}$ 821563 del caserío de Gallito Ciego del distrito de Yonán- Contumazá

\section{Tabla 1.}

Distribución de la población de estudiantes y docentes de educación primaria de la I.E. 821563-Gallito Ciego- Yonán, 2021.

\begin{tabular}{lccc}
\hline \multirow{2}{*}{ Participantes } & \multicolumn{2}{c}{ Sexo } & \multirow{2}{*}{ TOTAL } \\
\cline { 2 - 3 } Estudiantes & Hombres & Mujeres & 35 \\
Docentes & 16 & 19 & 3 \\
\hline \multicolumn{1}{c}{ TOTAL } & 0 & 3 & $\mathbf{3 8}$ \\
\hline
\end{tabular}

Nota. Base de datos ESCALE 2020. 
Los estudiantes son tanto varones como mujeres del primero al sexto grado, cuyas edades oscilan de seis a trece años, residentes en el área de influencia del centro educativo todos ellos hijos de padres de familia de situación socioeconómica media.

También participaron como población las docentes de los grados de estudio señalados anteriormente.

\subsection{Técnicas e instrumentos de recolección de datos}

La técnica empleada consistió en las encuestas plasmadas a través de un cuestionario respecto de las variables de estudio, aplicada a los alumnos, con 35 ítems dirigidos a las dimensiones correspondientes, fue redactado en base a interrogantes cerradas y se usó para su valoración la escala de Likert de tipo intervalo. De acuerdo a (López y Fachelli, 2015), señalan que el cuestionario es un instrumento para recopilar información por medio de la interrogación a las personas cuyo propósito es la obtención sistemáticamente medida respecto de los conceptos que devienen de un contexto problemático de investigación construida previamente.

Igualmente, se empleó para la recopilación de información en el trabajo de investigación la técnica de observación sistemática y espontánea en el salón de clase del estudiantado del sexto año de primaria mientras fue presencial y luego a través de los medios virtuales, así como la entrevista que posteriormente sirvió para organizar y sintetizar los resultados para dar una conclusión final.

\section{RESULTADOS}

Para los resultados se tendrán en cuenta los objetivos de investigación:

Tabla 2. Resultados del cuestionario aplicado a los estudiantes.

Niveles de las dimensiones del desarrollo del pensamiento crítico y creativo de los estudiantes de la institución educativa 821563-Gallito Ciego

\begin{tabular}{|l|c|c|c|c|c|c|c|}
\hline \multirow{2}{*}{ NIVEL } & \multicolumn{3}{|c|}{ DIMENSIONES PENSAMIENTO CRÍTICO } & \multicolumn{3}{c|}{ DIMENSIONES PENSAMIENTO } \\
\cline { 2 - 8 } & D. Lógica & D. Sustantiva & D. Dialógica & D. Contextual & D. Pragmática & D. de Problemas Movilidad \\
\hline Nivel Bajo & $82.90 \%$ & $71.40 \%$ & $82.90 \%$ & $88.60 \%$ & $77.10 \%$ & $65.70 \%$ & $82.90 \%$ \\
\hline Nivel Medio & $17.10 \%$ & $28.60 \%$ & $17.10 \%$ & $11.40 \%$ & $22.90 \%$ & $34.30 \%$ & $17.10 \%$ \\
\hline Nivel Alto & $0.00 \%$ & $0.00 \%$ & $0.00 \%$ & $0.00 \%$ & $0.00 \%$ & $0.00 \%$ & $0.00 \%$ \\
\hline
\end{tabular}

Nota. Base de datos del instrumento 


\section{Tabla 3.}

Niveles del desarrollo del pensamiento crítico y creativo de los estudiantes de la institución educativa 821563-Gallito Ciego

\begin{tabular}{ccc}
\hline \multirow{2}{*}{ NIVELES } & \multicolumn{2}{c}{ Pensamiento crítico y creativo } \\
\cline { 2 - 3 } & $\mathbf{f}$ & $\mathbf{\%}$ \\
\hline Bajo & 33 & 94.3 \\
Medio & 2 & 5.7 \\
Alto & 0 & 0 \\
\hline TOTAL & $\mathbf{3 5}$ & $\mathbf{1 0 0}$ \\
\hline
\end{tabular}

Nota. Base de datos del instrumento

En la Tabla 3 se evidencia que el $94.3 \%$ de los estudiantes actualmente posee un nivel bajo de pensamiento crítico y creativo, seguido del $5.7 \%$ que presenta un nivel medio y el $0 \%$ que presenta un nivel alto. Esto permite concluir que el pensamiento crítico y creativo de los estudiantes de la Institución Educativa 821563-Gallito Ciego-Cajamarca presenta un nivel mayoritariamente bajo, debido entre otras cosas a las deficientes estrategias usadas por los docentes para estimular el desarrollo del mismo.

Para determinar el grado de influencia que puede tener un programa de autorregulación emocional en el desarrollo del pensamiento crítico y creativo de los estudiantes de educación primaria se ha usado una encuesta, la misma que fue aplicada a los estudiantes con ayuda de los padres de familia, para de esta manera conocer el desarrollo actual del mismo. Asimismo, se proyecta un escenario con la posible aplicación del programa, que permite vislumbrar la utilidad del mismo de ser aplicado en las aulas de clase del nivel primario.

\section{Tabla 4.}

Tabla del desarrollo del pensamiento crítico y creativo de los estudiantes sin la aplicación del programa y con posible aplicación del programa

\begin{tabular}{ccccc}
\hline \multirow{2}{*}{ NIVELES } & Sin programa de autorregulación & \multicolumn{2}{c}{$\begin{array}{c}\text { Proyección con programa de } \\
\text { autorregulación }\end{array}$} \\
\cline { 2 - 5 } & $\mathbf{f}$ & $\mathbf{0}$ & $\mathbf{f}$ & $\mathbf{\%}$ \\
\hline Bajo & 33 & 94.3 & 0 & 0 \\
Medio & 2 & 5.7 & 1 & 2.9 \\
Alto & 0 & 0 & 34 & 97.1 \\
\hline TOTAL & $\mathbf{3 5}$ & $\mathbf{1 0 0}$ & $\mathbf{3 5}$ & $\mathbf{1 0 0}$ \\
\hline
\end{tabular}

Nota. Base de datos del instrumento 
En la Tabla 4 se evidencia que actualmente sin la aplicación del programa el 94.3\% de los estudiantes muestran un bajo nivel de pensamiento crítico y creativo; mientras que el $5.7 \%$ presentan un nivel medio y el $0 \%$ un nivel bajo. Contraria a esta situación, se evidencia que, con la eventual aplicación de programa de autorregulación, el 97.1\% de los estudiantes presentarían un nivel alto de pensamiento crítico y creativo, mientras que el $2.9 \%$ presentaría un nivel $2.9 \%$ y el $0 \%$ un nivel bajo. En conclusión, es apreciable la mejoría que se produciría en el desarrollo del pensamiento crítico mediante la aplicación de un programa de autorregulación, en consecuencia, resulta factible que dicha propuesta sea tomada en cuenta en posteriores estudios.

\section{DISCUSIÓN}

Los resultados en las dimensiones del pensamiento crítico y creativo permitieron concluir que el pensamiento crítico y creativo de los estudiantes de la Institución Educativa 821563-Gallito Ciego-Cajamarca presenta un nivel mayoritariamente bajo, debido entre otras cosas a las deficientes estrategias usadas por los docentes para estimular el desarrollo del mismo. En torno a estos resultados, se halla la investigación realizada por Freire, Guzmán, Cabrera y López (2019), en la cual concluyen que el pensamiento crítico, las habilidades de inteligencia emocional, y el desarrollo de capacidades para el aprendizaje influyen en el rendimiento académico mejorando la comprensión, la comunicación y la resolución de situaciones problemáticas que mejoran la calidad de vida en entornos educativos, familiares y sociales. Se deben potenciar las particularidades de la inteligencia emocional, empatía, autoconciencia, adaptabilidad, autorregulación, iniciativa, habilidades sociales, liderazgo comunicación, y trabajo en equipo, con la expectativa de que contribuyan al desarrollo cognitivo y mejoren los resultados del aprendizaje.

Se evidencia que actualmente sin la aplicación del programa los estudiantes muestran un bajo nivel de pensamiento crítico y creativo. Contraria a esta situación, se evidencia que, con la eventual aplicación de programa de autorregulación, los estudiantes presentarían un nivel alto de pensamiento crítico y creativo. En conclusión, es apreciable la mejoría que se produciría en el desarrollo del pensamiento crítico mediante la aplicación de un programa de autorregulación, en consecuencia, resulta factible que dicha propuesta sea tomada en cuenta en posteriores estudios. Los resultados expuestos guardan relación con el trabajo desarrollado por Coello y Cepeda (2018), en el mismos que concluyen que el 
estudiantado, aunque conscientes de los elementos positivos de la inteligencia emocional, muestran limitaciones y falta de comprensión de los diferentes aspectos de su interés, por lo que no se puede decir que comprendan lo ventajoso de la inteligencia emocional. Los alumnos no están familiarizados con las diversas técnicas de inteligencia emocional que les ayudan a afianzar sus habilidades de pensamiento crítico. Por ello, con la posible aplicación del programa se producirán mejoras en el desarrollo del pensamiento creativo y crítico en los alumnos, debido a las actividades y sesiones que se realizarán y que permitirán ir modulando y evaluando los progresos de cada uno de ellos.

Respecto de la hipótesis general, esta se comprueba puesto que el programa de autorregulación emocional contribuye al desarrollo significativo del pensamiento crítico y creativo de los estudiantes puesto que un estudiante que aprende a controlar sus emociones, impulsos o acciones será capaz de tomar una actitud crítica frente a condiciones desencadenantes o estímulos relevantes; y a su vez hallar salidas creativas y variadas al problema que se presente. Teóricamente, la hipótesis tiene sustento en los aportes de Melamed, Ennis, Akaydin y Akduman; la necesidad de posibilitar que, en los centros educativos, los estudiantes tengan espacios de pensamiento en los que puedan participar de manera activa, crítica fomenta la apertura. Para solucionar problemáticas complejas y para crear innovaciones se precisa la mezcla de la inventiva con el pensamiento crítico.

La validación de la propuesta fue llevada a cabo por el juicio de expertos obteniendo la valoración de bastante adecuada, quedando establecido que la misma se halla adecuadamente construida y puede ser aplicada en posteriores estudios a realizarse. Finalmente, bajos los supuestos explicados y con la validación respectiva, se concluye que el programa de autorregulación emocional se erige en un elemento primordial, que en cuanto se aplique genera el desarrollo significativo del pensamiento crítico y creativo en los estudiantes de la institución educativa 821563-Gallito Ciego, quedando de esta manera demostrada la hipótesis de estudio propuesta.

\section{CONCLUSIONES}

Se debe señalar que es rol principal de la escuela preparar seres humanos íntegros, que aporten a la construcción de sociedad; en consecuencia, es de crucial relevancia aplicar novedosas estrategias que permitan mejorar el desarrollo de pensamiento crítico y creativo, permitiendo a los niños actuar de forma independiente y autónoma en ese 
proceso de construcción. Por tanto, es preciso trabajar desde el salón novedosas metodologías que involucren para el estudiantado la toma adecuada de determinaciones ante distintas situaciones, mediante el auto reconocimiento, percibiendo sus emociones propias y las de los demás, y que, de similar manera, les posibilite procedimientos de inferencia, análisis, definición de pautas, y en función a estos tengan la posibilidad de innovar, crear, construir, su inteligencia emocional y su pensamiento crítico y creativo. Este estudio pretende ser un aporte para los docentes en el manejo de las emociones y la formación del pensamiento crítico y creativo del estudiantado de educación primaria, a través de la propuesta desarrollada se presenta una serie de actividades y sesiones educativas que buscan contribuir en la resolución de problemas que pudieran presentarse en el contexto educativo y social del estudiantado, permitiendo que por medio de esto puedan llegar a participar de una transformación comunitaria positiva en el ambiente en donde se encuentran inmersos. 
5. PROPUESTA: Modelo del Programa de autorregulación emocional para el pensamiento crítico y creativo en estudiantes de la institución educativa 821563-Gallito Ciego

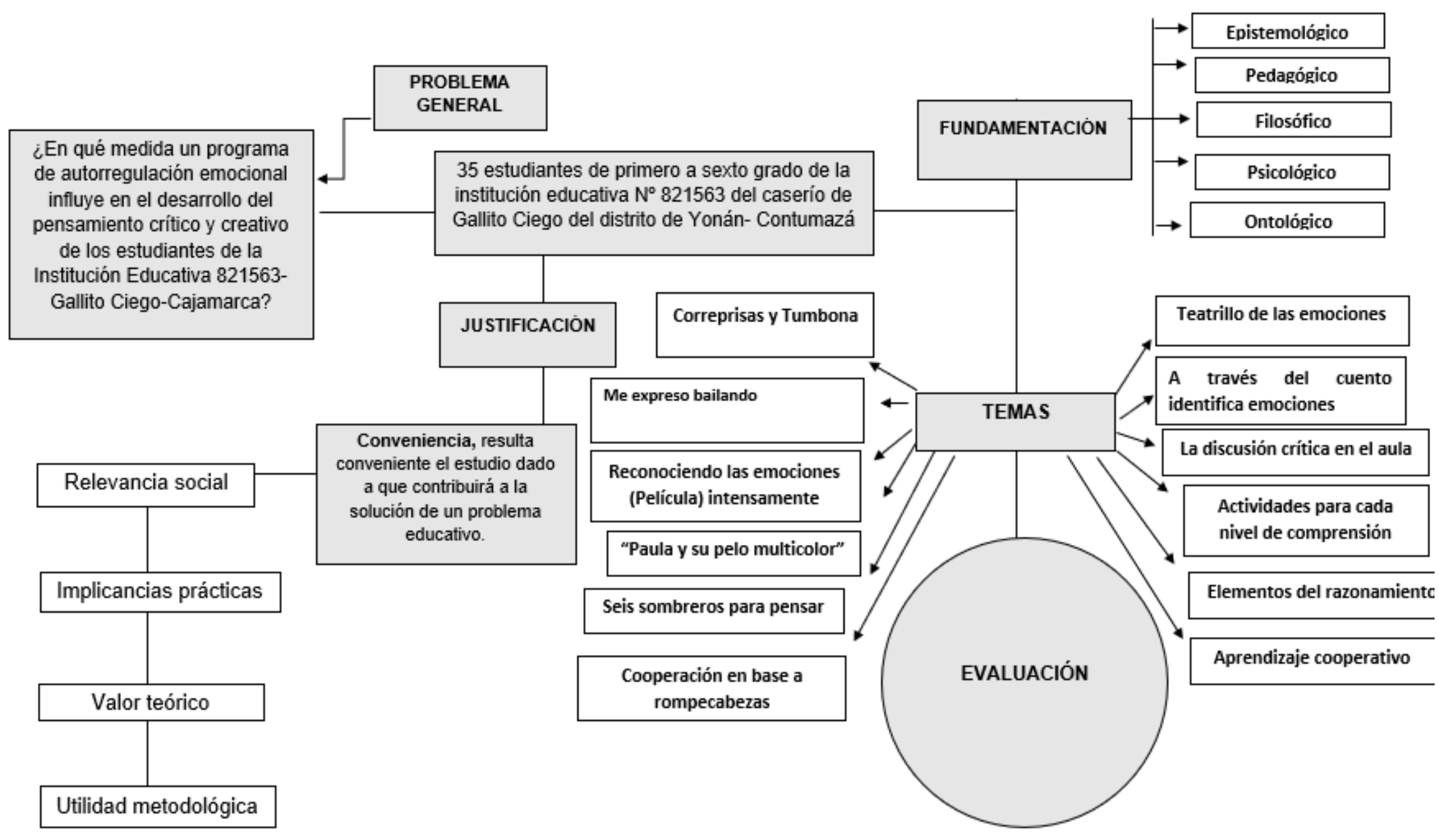

Ciencia Latina Revista Científica Multidisciplinar, Ciudad de México, México.

ISN 2707-2207 / ISSN 2707-2215 (en línea), enero- febrero, 2022, Volumen 6, Número 1. https://doi.org/10.37811/cl_rcm.v6i1.1545 p845 


\section{REFERENCIAS BIBLIOGRAFICAS}

Akaydin, D. y Akduman, G. (2016). Emotional Intelligence in Children. Current Advances in Education (pp. 347-354).

Álvarez, A. (2020). Clasificación de las Investigaciones. Universidad de Lima.

Coello, M. y Cepeda, C. (2018). Inteligencia emocional en desarrollo del pensamiento crítico. Taller técnicas de control emocional [Tesis de grado]. Universidad de Guayaquil. http://repositorio.ug.edu.ec/bitstream/redug/44735/1/BFILO-PD-LP118-053.pdf

Freire, J., Guzmán, B., Cabrera, J. y López, M. (2019). Inteligencia emocional: una competencia para fortalecer el desarrollo cognitivo, el pensamiento crítico, la toma de decisiones y el rendimiento académico. Ciencia Digital, 3(2), 645-659. https://doi.org/10.33262/cienciadigital.v3i2.472

Giler, J. (2018). Investigación Diagnóstica o Propositiva, Método científico, Investigación cualitativa.

Guevara, G., Verdesoto, A. y Castro, N. (2020). Metodologías de investigación educativa (descriptivas, experimentales, participativas, y de investigación-acción). Recimundo Vol. 4 (3). DOI: 10.26820/recimundo/4.(3).julio.2020.163-173

Hernández, R., Fernández, C., y Baptista, P. (2014). Metodología de la investigación. McGraw-Hill.

Lafourcade, M. (2018). Seis dimensiones de una mente creativa. La teoría disposicional. Editorial basilisa.

Larraz, N. (2020). La competencia emocional e interpersonal en el ámbito educativo. Universidad de Zaragoza. https://zaguan.unizar.es/record/94466/files/BOOK-2020126.pdf

Lazo, L. y Herrera, H. (2011). Aplicación de un modelo de intervención pedagógica que desarrolla estrategias de pensamiento crítico para estudiantes de carreras del área de las ciencias. Revista electrónica diálogos educativos, 21 (11). http://www.umce.cl/ dialogos/n21_2011/lazo.swf

López, H., Talavera, J. y Rodríguez, O. (2020). Incidencia del ejercicio físico en la regulación emocional de los estudiantes de undécimo grado A del Instituto Guillermo Cano Balladares, de la ciudad de Estelí, en el I semestre del año 2019. [Tesis de grado]. Universidad nacional Autónoma de Nicaragua, Managua. 
Lopez, P. y Fachelli, S. (2015). Metodología de la investigación social cuantitativa. Universitat Autònoma de Barcelona.

Lubart, T. (2018). Creativity through the seven cs. The nature of human creativity. The nature of human creativity, 134-145.

Luján, C. (2019). Inteligencia emocional y pensamiento crítico en estudiantes de $4 .^{\circ}$ y 5 . $^{\circ}$ de secundaria de un colegio privado de Jesús María - 2019. Universidad César Vallejo [Tesis de maestría]. https://repositorio.ucv.edu.pe/bitstream/handle/20.500.12692/41647/Luj\%c3\% a1n_MC.pdf?sequence $=1 \&$ isAllowed $=\mathrm{y}$

Melamed, A. (2016). Las teorías de las emociones y su relación con la cognición: un análisis desde la filosofía de la mente. Cuadernos de la Facultad de Humanidades y Ciencias Sociales, núm. 49.

Ministerio de Educación. (2015a). Evaluación PISA 2015. http://umc.minedu.gob.pe/resultados-de-evaluacion-pisa-2015/

Restrepo, C. y Saavedra, L. (2020). Autorregulación de emociones una propuesta de intervención psico-educativa en la Institución Educativa "La Fontaine". [Tesis de grado], Universidad Cooperativa de Colombia.

Rocha, C. (2015). Metodología de la investigación. Oxford University Press

Rodríguez, A. (2019). Pensamiento Crítico: Características, Habilidades y Actitudes. https://www.lifeder.com/pensamiento-critico/

Salazar, B. (2019). Las TIC en la educación: una enseñanza más activa e innovadora. Universidad de Piura. https://www.udep.edu.pe/hoy/2019/07/las-tic-en-laeducacion-una-ensenanza-mas-activa-e-innovadora/

Sineace (2020). Caracterización de la región Cajamarca 2020. Ministerio de Educación. Unesco (2019). Estudio Regional Comparativo y Explicativo (ERCE). Tercer estudio Regional comparativo y explicativo (Terce). 